Pacific Journal of Mathematics

CONVERGENCE OF ADAPTED SEQUENCES O 


\section{CONVERGENCE OF ADAPTED SEQUENCES OF PETTIS-INTEGRABLE FUNCTIONS}

\section{EGGHE}

When considering adapted sequences of Pettis-integrable functions with values in a Banach space we are dealing with the following problem: when do we have a strongly measurable Pettis-integrable limit? Here the limit can be taken in the strong or weak sense a.e. or in the sense of the Pettis-topology.

Not many results in this area are known so far.

In this paper we give some pointwise convergence results of martingales, amarts, weak sequential amarts and pramarts consisting of strongly measurable Pettis-integrable functions. Also the Pettis convergence result of Musial for amarts is extended.

The results are preceded by a preliminary study of some vector measure notions such as Pettis uniform integrability and $\sigma$-bounded variation. We give a new proof of the result of Thomas stating that in every infinite dimensional Banach space one can find a vector measure which is not of $\sigma$-bounded variation.

1. Introduction, terminology and notation. In the sequel, $E$ will be a Banach space and $(\Omega, \mathscr{F}, P)$ a fixed complete probability space. A function $X: \Omega \rightarrow E$ is called scalarly measurable if $\left\langle x^{\prime}, X\right\rangle$ is measurable for each $x^{\prime} \in E^{\prime}$.

A function $X$ is called Pettis-integrable if it is scalarly integrable and if for each $A \in F$, there exists $x_{A} \in E$ such that, for each $x^{\prime} \in E^{\prime}$

$$
\left\langle x^{\prime}, x_{A}\right\rangle=\int_{A}\left\langle x^{\prime}, X\right\rangle d P .
$$

$x_{A}$ is denoted by $\int_{A} X d P$, the Pettis-integral of $X$ over $A$. Let $X$ and $Y$ be two Pettis-integrable functions. We say that $X$ is weakly equivalent with $Y$, denoted by $X \sim Y$, if for each $x^{\prime} \in E^{\prime}$

$$
\left\langle x^{\prime}, X\right\rangle=\left\langle x^{\prime}, Y\right\rangle \text {, a.e. }
$$

Denote by $P_{E}$ the space of all Pettis-integrable functions up to weak equivalence. Put on $P_{E}$ the following norm, called Pettis-norm,

$$
\|\cdot\|_{P}: P_{E} \rightarrow R^{+}, \quad X \rightarrow\|X\|_{P}=\sup _{\substack{\left\|x^{\prime}\right\| \leq 1 \\ x^{\prime} \in E^{\prime}}} \int_{\Omega}\left|\left\langle x^{\prime}, X\right\rangle\right| d P .
$$


The notions of strong measurability and Bochner-integrability we suppose to be familiar to the reader (see [D-U]).

It is trivial that every Bochner-integrable function is Pettis-integrable and that mean convergence (i.e. for the norm $\|\cdot\|_{1}$ on $L_{E}^{1}$, the space of Bochner-integrable functions) implies $\|\cdot\|_{P}$ convergence. The converse of these two implications is never true except in finite dimensional spaces as is well known. Even if we suppose that every strongly measurable Pettis-integrable function is Bochner-integrable we can prove that $E$ must be finite dimensional. Indeed, suppose $E$ is infinite dimensional. Using the theorem of Dvoretzky-Rogers (see [D-R]) there exists an unconditionally convergent series $\sum x_{n}$ such that $\sum\left\|x_{n}\right\|=\infty$. Let $\left(A_{n}\right)_{n \in N}$ be a countable measurable partition of $\Omega$ such that $P\left(A_{n}\right)>0$ for each $n \in N$. Put

$$
X=\sum_{n \in N} \frac{x_{n}}{P\left(A_{n}\right)} \chi_{A_{n}} .
$$

Then $X$ is Pettis-integrable strongly measurable and not Bochner-integrable. Of course here one has $\|X\|_{1}=\infty$ and $\|X\|_{P}<\infty$.

Let $\left(\mathscr{F}_{n}\right)_{n \in N}$ be a stochastic basis, i.e. an increasing sequence of sub- $\sigma$-algebras of $\mathscr{F}$. Let $\left(X_{n}\right)_{n \in N}$ be a sequence in $P_{E}$. We say that $\left(X_{n}, \mathscr{F}_{n}\right)_{n \in N}$ is an adapted sequence if each $X_{n}$ is $\mathscr{F}_{n}$-scalarly measurable. If in addition each $X_{n}$ is strongly measurable then $X_{n}$ is $\mathscr{F}_{n}$-strongly measurable as follows easily from Pettis' measurability theorem (see [D-S]). With respect to a stochastic basis $\left(\mathscr{F}_{n}\right)_{n \in N}$, we say that a function $\tau: \Omega \rightarrow N$ is a stopping time if $\{\tau=n\} \in \mathscr{F}_{n}$ for each $n \in N$.

Let us denote by $T$ the set of all bounded (i.e. finitely valued) stopping times. We order $T$ in the natural (pointwise) way. For $\tau \in T$ and $\left(X_{n}, \mathscr{F}_{n}\right)_{n \in N}$ an adapted sequence, define

$$
X_{\tau}=\sum_{k=\min \tau}^{\max \tau} X_{k} \chi_{\{\tau=k\}} .
$$

Also define $\mathscr{F}_{\tau}=\left\{A \in \mathscr{F} \mid A \cap\{\tau=k\} \in \mathscr{F}_{k}\right.$ each $\left.k \in N\right\}$.

It is obvious that $X_{\tau}$ is scalarly $\mathscr{F}_{\tau}$-measurable. Adapted sequences of Bochner-integrable functions have been studied intensively. We refer to [Eg] which is a monograph on this subject which is about to appear at this moment. On the contrary, on adapted sequences of Pettis-integrable functions virtually nothing is known. This is of course due to the completely different nature of the Pettis-integral (see remark above). To illustrate this see also $\left[\mathbf{T}_{3}\right],\left[\mathbf{M} \mathbf{u}_{1}\right],\left[\mathbf{M} \mathbf{u}_{\mathbf{2}}\right]$. Before mentioning the results, let us give some well-known definitions. Let $\left(X_{n}, \mathscr{F}_{n}\right)_{n \in N}$ be an adapted sequence in $P_{E}$. 
It is called a martingale if for each $n \in N$ and each $A \in \mathscr{F}_{n}$ :

$$
\int_{A} X_{n}=\int_{A} X_{n+1}
$$

(Here the integrals are in the Pettis sense of course.) In other words, $X_{n}=\mathscr{E}^{\mathscr{F}_{n}} X_{n+1}$, where $\mathscr{E}^{\mathscr{F}_{n}}$ denotes the Pettis conditional expectation w.r.t. $\mathscr{F}_{n}$. It must be emphasized that the Pettis conditional expectation of a Pettis integrable function does not always exist, even if the function is strongly measurable; see $\left[\mathbf{M u}_{\mathbf{1}}\right]$ and also $§ 2$.

Of course, as is well-known and easy to prove, the conditional expectation of a Bochner integrable function always exists.

An adapted sequence $\left(X_{n}, \mathscr{F}_{n}\right)_{n \in N}$ is called an amart if the net $\left(\int_{\Omega} X_{\tau}\right)_{\tau \in T}$ converges in $E$. It is obvious that every martingale is an amart. Finally a Banach space $E$ is said to have the weak Radon-Nikodym-property (abbreviated (WRNP)) if for every complete probability space $(\Omega, \mathscr{F}, P)$ and for every vector measure $F: \mathscr{F} \rightarrow E$ of $\sigma$-bounded variation which is $P$-continuous, there exists $X \in P_{E}$ such that for each $A \in \mathscr{F}$ :

$$
F(A)=\int_{A} X
$$

An equivalent statement of (WRNP) is obtained if one replaces " $\sigma$ bounded variation" by "bounded variation".

However the first definition is more natural since a Pettis integral is always of $\sigma$-bounded variation.

For a discussion of (WRNP) we refer the reader to $\left[\mathbf{M u} \mathbf{u}_{1}\right]$ or $\left[\mathbf{M u} \mathbf{u}_{2}\right]$.

The first result we have to mention concerning convergence of adapted sequences of strongly measurable Pettis integrable functions is one of J. J. Uhl Jr.:

TheOREM ([ $\left.\mathbf{U}_{2}\right]$, THEOREM 3.1): Let $\left(X_{n}, \mathscr{F}_{n}\right)_{n \in N}$ be a martingale consisting of strongly measurable Pettis-integrable functions.

Suppose

(i) $\sup _{n \in N}\left\|X_{n}\right\|_{P}<\infty$.

(ii) The set $\left\{\int_{A} X_{n} \mid A \in \mathscr{F}_{n}, n \in N\right\}$ is weakly relatively compact.

(iii) For each $\varepsilon>0$ there is a weakly compact set $K \subset E$ such that for each $\delta>0$, there exists $n_{0} \in N$ and $A_{0} \in \mathscr{F}_{n_{0}}$ for which $P\left(\Omega \backslash A_{0}\right)<\varepsilon$ such that $n \geq n_{0}$ implies $\int_{A} X_{n} \in P(A) K+\delta B_{E}$ for all $A \subset A_{0}, A \in \mathscr{F}_{n}$ (here $\left.B_{E}=\{x \in E \mid\|x\| \leq 1\}\right)$.

Then $\left(X_{n}\right)_{n \in N}$ converges strongly a.e. to a function in $P_{E}$. 
Condition (iii) is a Rieffel-type condition (see e.g. [Die] Theorem 1, pp. 204-205) and is satisfied if $E$ has (RNP) and if (i) is strengthened to $\sup _{n \in N}\left\|X_{n}\right\|_{1}<\infty$, in which case we have the Chatterji results on martingales consisting of Bochner integrable functions (see [C]) (condition (ii) can be dropped in this case; see Corollary 3.4 in $\left.\left[\mathbf{U}_{2}\right]\right)$. So this is an interesting and nontrivial extension of the classical martingale convergence theorem.

Concerning weak convergence we refer to [Me]; we are not dealing with weak convergence of martingales in this paper.

Another result - one of Musial - can be stated as follows:

THEOREM ([Mu $]$, THEOREM 5). The following assertions are equivalent:

(i) $E$ has (WRNP).

(ii) For every complete probability space $(\Omega, \mathscr{F}, P)$ and every martingale $\left(X_{n}, \mathscr{F}_{n}\right)_{n \in N}$ in $P_{E}$ on $(\Omega, \mathscr{F}, P)$ which is Pettis uniformly integrable and variationally bounded there is an $X \in P_{E}$ such that $\lim _{n \rightarrow \infty}\left\|X_{n}-X\right\|_{P}=0$.

Here, Pettis uniform integrability means

$$
\lim _{P(A) \rightarrow 0} \sup _{n \in N}\left\|\int_{A} X_{n}\right\|=0 .
$$

See also $\$ 2$. Variationally bounded means that

$$
\sup _{n \in N}\left|\nu_{n}\right|(\Omega)<\infty
$$

where $\left|\nu_{n}\right|$ denotes the variation of

$$
\nu_{n}: \mathscr{F}_{n} \rightarrow E, \quad A \rightarrow \int_{A} X_{n} .
$$

The extension to amarts in the above theorem can easily be made in Musial's proof.

However, Musials' theorem, when stated for (RNP) spaces teaches nothing new, even when it is stated for amarts. Indeed, in this case, as is easily seen (see e.g. [J], p. 129), for each $n \in N$, there exists $Y_{n} \in P_{E}$, strongly measurable such that $Y_{n} \sim X_{n}$. From the definition of variation and from $Y_{n} \sim X_{n}$ for each $n \in N$ it now follows that

$$
\sup _{n \in N} \int_{\Omega}\left\|Y_{n}\right\|=\sup _{n \in N} \int_{\Omega}\left\|X_{n}\right\|<\infty .
$$


So we have here an $L_{E}^{1}$-bounded amart satisfying also

$$
\lim _{P(A) \rightarrow 0} \sup _{n \in N}\left\|\int_{A} Y_{n}\right\|=0 \text {. }
$$

This is a known situation ([ $\left.\mathbf{U}_{3}\right]$, Corollary 4, p. 294) yielding a Bochner integrable function $Y$ such that

$$
\lim _{n \rightarrow \infty}\left\|Y_{n}-Y\right\|_{P}=0 \text {. }
$$

But since $X_{n} \sim Y_{n}$ for each $n \in N$ we also have

$$
\lim _{n \rightarrow \infty}\left\|X_{n}-Y\right\|_{P}=0 \text {. }
$$

So, Musial's theorem reduces - in case $E$ has (RNP) - to Uhl's theorem $\left[\mathbf{U}_{3}\right]$, Corollary 4 , p. 294 , and is certainly only a convergence theorem for amarts consisting of Bochner integrable functions.

However, it would be interesting to have convergence results in (RNP) Banach spaces for strongly measurable Pettis-integrable amarts and martingales which are not Bochner integrable. This is one of the purposes of this article. The paper is divided as follows: In $\$ 2$ we study the notions $\sigma$-bounded variation and Pettis-uniform integrability. Concerning the first notion a result of Thomas [ $\mathbf{T}_{2}$ ] (later reproved by Janicka-Kalton [ $\mathbf{J}-\mathbf{K}]$ ) is reproved yielding a straightforward proof. In $\S 3$ we prove a martingale strong a.e. convergence theorem for strongly measurable functions in $P_{E}$ where $E$ has (RNP).

Section 4 reproves and generalizes the Pettis convergence theorems for amarts of Musial and Uhl. A Riesz decomposition theorem is proved for amarts in $P_{E}$ generalizing the one of Edgar-Sucheston.

Section 5 proves a weak a.e. convergence theorem for weak sequential amarts.

Finally $\$ 6$ proves a strong a.e. convergence result for pramarts consisting of strongly measurable functions in $P_{E}$ where $E$ has (RNP), extending a theorem of Millet-Sucheston.

\section{Study of some notions related to the Pettis integral.}

\subsection{Vector measures of $\sigma$-bounded variation.}

Definition 2.1.1. Let $F: \mathscr{F} \rightarrow E$ be a vector measure. We say that $F$ is of $\sigma$-bounded variation if there is a disjoint sequence $\left(A_{n}\right)_{n \in N}$ in $\mathscr{F}$ such that the restriction $\left.F\right|_{A_{n}}$ to $A_{n}$ is a vector measure of bounded variation, for each $n \in N$. 
This is a notion which is very natural in connection with Pettis integrals. Indeed we have that every Pettis integral (considered as a vector measure) is of $\sigma$-bounded variation. This is a result of Musial, see [ $\left.\mathbf{M u} \mathbf{u}_{\mathbf{1}}\right]$. Compare this with the well known fact that every Bochner integral is a vector measure of bounded variation. As is the case with the Pettis integral, the notion of $\sigma$-bounded variation is not easy to study. For instance, if $F: \mathscr{F} \rightarrow E$ is of bounded variation and if $\mathscr{G}$ is a sub- $\sigma$-algebra of $\mathscr{F}$ then it is completely trivial that $F \mid \mathscr{G}$ is of bounded variation. This is false for the $\sigma$-bounded variation notion. This is easy to prove and firstly remarked by Musial $\left[\mathbf{M} \mathbf{u}_{\mathbf{1}}\right]$. Indeed we have:

Proposition 2.1.2. Suppose $E$ has (WRNP). Let $F: \mathscr{F} \rightarrow E$ be given by a Pettis integral

$$
F(A)=\int_{A} X
$$

for each $A \in \mathscr{F}$, and let $\mathscr{G}$ be a sub- $\sigma$-algebra of $\mathscr{F}$. The $F \mid \mathscr{G}$ is of $\sigma$-bounded variation iff $E^{\mathscr{G}} X$ exists in $P_{E}$.

Proof. The proof is very easy and included for the sake of completeness. Indeed, suppose $F \mid \mathscr{G}$ is of $\sigma$-bounded variation.

Since $F \ll P \mid \mathscr{G}$ we have also $F|\mathscr{G} \ll P| \mathscr{G}$.

So, using (WRNP), there exists a function $Y \in P_{E}(\Omega, \mathscr{G}, P \mid \mathscr{G})$ such that for each $A \in \mathscr{G}$

$$
(F \mid \mathscr{G})(A)=\int_{A} Y
$$

By the form of $F, Y$ must be $E^{\mathscr{G}} X$.

Conversely, suppose that $E^{\mathscr{G}} X \in P_{E}(\Omega, \mathscr{G}, P \mid \mathscr{G})$ exists. Then for each $A \in \mathscr{G}$

$$
(F \mid \mathscr{G})(A)=\int_{A} E^{\mathscr{G}} X
$$

and since this is a Pettis integral, it is of $\sigma$-bounded variation (see $\left.\left[\mathbf{M u}_{1}\right]\right)$.

Now it is clear that a vector measure $F$ of $\sigma$-bounded variation (w.r.t. a $\sigma$-algebra $\mathscr{F}$ ) exists such that $F \mid \mathscr{G}$ is not of $\sigma$-bounded variation, where $\mathscr{G}$ is a sub- $\sigma$-algebra of $\mathscr{F}$. It suffices to take a Pettis integrable function $f$ : $\mathscr{F} \rightarrow E$ without a conditional expectation. see $\left[\mathbf{M u _ { 1 }}\right],[\mathbf{H}],[\mathbf{R}]$. 
Although Proposition 2.1.2 is interesting it does not solve completely the problem of when a vector measure is of $\sigma$-bounded variation w.r.t. a sub- $\sigma$-algebra.

Another question one might ask is the following: Suppose a vector measure $F$ is absolutely continuous w.r.t. Lebesgue-measure on $[0,1]$. Is $F$ of $\sigma$-bounded variation?

The property is obviously true in any finite dimensional Banach space. This was disproved in any infinite dimensional Banach space by $\mathrm{G}$. Thomas in $\left[\mathbf{T}_{\mathbf{2}}\right]$ and later by Janicka-Kalton $[\mathbf{J}-\mathbf{K}]$. Incidently $\mathbf{I}$ found a new proof on this fact in a very straightforward way. It runs as follows:

THEOREM 2.1.3 (Thomas). Suppose $E$ is an infinite dimensional Banach space. Then there exists a probability space $(\Omega, \mathscr{F}, P)$ and a vector measure $F: \mathscr{F} \rightarrow E$ such that $F \ll P$ and such that $F$ is not of $\sigma$-bounded variation.

Proof. The characterization of infinite dimensional Banach spaces of Dvoretzky-Rogers [D-R] yields a sequence $\left(x_{n}\right)_{n \in N}$ in $E$ such that $\sum x_{n}$ converges unconditionally and such that $\sum\left\|x_{n}\right\|=\infty$.

Put $x_{0}=\sum_{n=1}^{\infty} x_{n}$.

Write

$$
\begin{aligned}
& x_{1}=\left(x_{1}-x_{0}\right)+\sum_{n=1}^{\infty} x_{n}=\sum_{n=1}^{\infty}\left[\frac{1}{2^{n}}\left(x_{1}-x_{0}\right)+x_{n}\right] \\
& x_{2}=\left(x_{2}-x_{0}\right)+\sum_{n=1}^{\infty} x_{n}=\sum_{n=1}^{\infty}\left[\frac{1}{2^{n}}\left(x_{2}-x_{0}\right)+x_{n}\right] .
\end{aligned}
$$

Then do the same with the first term of the series appearing in (1), then with the second and so on. The same with (2) and so on. Every series appearing above converges unconditionally but not absolutely since (we take the series in (1) - an analogous proof for the other series)

$$
\begin{aligned}
\sum_{n=1}^{\infty}\left\|\frac{1}{2^{n}}\left(x_{1}-x_{0}\right)+x_{n}\right\| & \geq \sum_{n=1}^{\infty}\left|\left\|x_{n}\right\|+\frac{1}{2^{n}}\left\|x_{1}-x_{0}\right\|\right| \\
& \geq \sum_{n=1}^{\infty}\left\|x_{n}\right\|-\left\|x_{1}-x_{0}\right\|=\infty
\end{aligned}
$$


Let now $\sum y_{n}$ be any of the series introduced above. By assuming $x_{0} \neq 0$ and $x_{n} \neq 0$ for each $n \in N$ and by making additions and relabelings we can assume that

$$
y_{n} \neq 0, \quad \forall n \in N \quad \text { and } \quad\left\|\sum_{n=1}^{\infty} y_{n}\right\|>2\left\|y_{k}\right\|, \quad \forall k \geq 2 .
$$

(Since $\sum_{k=1}^{n} y_{k} \rightarrow \sum_{n=1}^{\infty} y_{n}$ and since $y_{n} \rightarrow 0$ and $\left\|\sum_{n=1}^{\infty} y_{n}\right\|>0$ there exists $n_{0} \in N$ such that $\left\|\sum_{k=1}^{n_{0}} y_{k}\right\|>2\left\|y_{i}\right\|, \forall i>n_{0}$. Relabel $y_{i}^{\prime}=\sum_{k=1}^{n_{0}} y_{k}, y_{2}^{\prime}=$ $y_{n_{0}+1}$, and so on.)

Take now $] 0,1]$ and divide it dyadically into

$$
] \frac{1}{2}, 1\right],\right] \frac{1}{4}, \frac{1}{2}\right],\right] \frac{1}{8}, \frac{1}{4}\right], \ldots
$$

Each of these intervals are also divided dyadically in the same way (homothetically). This constitutes the second level. The same for the third level and so on.

We are now going to construct the vector measure $F$.

We put the following values for the first level intervals

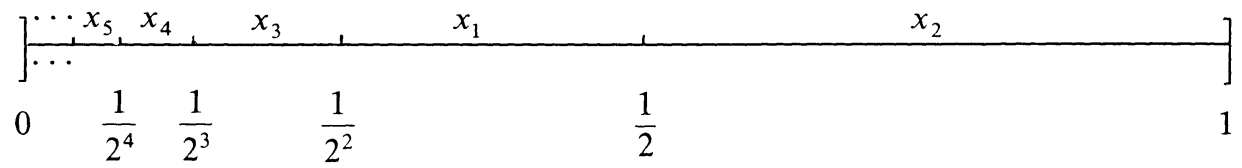

i.e. $x_{l}$ on $\left.] 1 / 2^{i}, 1 / 2^{i-1}\right]$ except for $x_{1}$ and $x_{2}$ or: We put first $x_{1}$ on the second place from the right and then put the vectors $x_{2}, x_{3}, x_{4}, \ldots$ consecutively from the right on the not yet used intervals. Let $\sum y_{n}$ be one of the series of the second level adding up to, say $x_{i}$. Here we put on the interval where we have put the value $x_{i}$ :

\begin{tabular}{lllll}
$\cdots, y_{5}, y_{4}$ & $y_{1}$, & $y_{3}$ & $y_{2}$ \\
\hline$\frac{1}{2^{5}} \frac{1}{2^{4}} \frac{1}{2^{3}}$ & $\frac{1}{2^{2}}$ & $\frac{1}{2}$ &
\end{tabular}

(homothetic interval and divisions)

$y_{1}$ on the third place from the right (division $1 / 2^{3}$ relatively to the interval) and then put the vectors $y_{2}, y_{3}, y_{4}, \ldots$ consecutively from the right on the not yet used intervals.

Inductively, let $\sum y_{n}$ be one of the series from the $n$th level. Here, we put $y_{1}$ on the $(n+1)$ th place from the right (division $1 / 2^{n+1}$ relatively to the interval in consideration) and then put the vectors $y_{2}, y_{3}, y_{4}, \ldots$, consecutively from the right on the not yet used intervals. 
Now we estimate from above the total length of the intervals on which the first terms $y_{1}$ are appearing.

For the first level: just one place: length $1 / 2^{2}$.

For the second level:

$$
\frac{1}{2} \cdot \frac{1}{2^{3}}+\frac{1}{4} \cdot \frac{1}{2^{3}}+\cdots=\sum_{n=1}^{\infty} \frac{1}{2^{n}} \frac{1}{2^{3}}=\frac{1}{2^{3}}
$$

For the fourth level: $1 / 2^{4}$ and so on.

A majorization is obtained by adding these values: $\sum_{n=2}^{\infty} 1 / 2^{n}=1 / 2$ (considering all the intervals on which the $y_{1}$ are appearing as disjoint which is not the case). Call the complement of this set $A$. So $\lambda(A) \geq \frac{1}{2}$ ( $\lambda=$ Lebesgue measure).

Call $F$ the constructed vector measure, restricted to $A$. Since on $A$, every vector appearing in the $n$th level has norm smaller than $\frac{1}{2}$ times the norm of the corresponding vector in the $(n-1)$ th level, we have that $F$ on $A$ is $\left.\lambda\right|_{A^{-}}$continuous.

Also since for every series $\sum y_{n}$, appearing in the construction we have that $\sum\left\|y_{n}\right\|=\infty$ it follows that $F$ is not of $\sigma$-bounded variation.

We end with a problem of Musial $\left[\mathbf{M u}_{\mathbf{1}}\right]$ which would (in case of an affirmative solution) completely settle the problem concerning $\sigma$-bounded variation w.r.t. a sub- $\sigma$-algebra as well as extend the result of Thomas (Theorem 2.1.3.)

Problem 2.1.4. Is it true that in any infinite dimensional Banach space $E$ there exists a probability space $(\Omega, \mathscr{F}, P)$, a $P$-continuous measure $F$ : $\mathscr{F} \rightarrow E$ of $\sigma$-bounded variation and a sub- $\sigma$-algebra $\mathscr{G}$ of $\mathscr{F}$ such that $F \mid \mathscr{G}$ is not of $\sigma$-bounded variation?

2.2. Pettis uniform integrability. If $\left(X_{n}\right)_{n \in N}$ is a sequence in $L_{E}^{1}$ two well-known equivalent definitions of uniform integrability are in use:

(i) $\sup _{n \in N}\left\|X_{n}\right\|_{1}<\infty$ and $\lim _{P(A) \rightarrow 0} \sup _{n \in N} \int_{A}\left\|X_{n}\right\|=0$.

(ii) $\lim _{\lambda \rightarrow \infty} \sup _{n \in N} \int_{\left\{\left\|X_{n}\right\|>\lambda\right\}}\left\|X_{n}\right\|=0$. Obviously, $L_{E}^{1}$-norms are involved here. When working with sequences $\left(X_{n}\right)_{n \in N}$ in $P_{E}$ the following definition of Pettis uniform integrability is quite natural.

Definition 2.2.1. The sequence $\left(X_{n}\right)_{n \in N}$ is said to be Pettis uniformly integrable if

$$
\sup _{n \in N}\left\|X_{n}\right\|_{P}<\infty \quad \text { and } \quad \lim _{P(A) \rightarrow 0} \sup _{n \in N} \sup _{B \in F}\left\|\int_{A \cap B} X_{n}\right\|=0 .
$$


Indeed the Pettis norm is involved here since the norm $\sup _{A \in \mathscr{F}}\left\|\int_{A} X_{n}\right\|$ is equivalent with $\left\|X_{n}\right\|_{P}$.

Definition 2.2.1 is not only natural because of the replacement of $\|\cdot\|_{1}$ by $\|\cdot\|_{P}$ in the classical definition of uniform integrability. It is also a "uniform" notion of Pettis integrability. Indeed, in $[\mathbf{P}]$ it is proved that a Pettis integrable function satisfies

$$
\lim _{P(A) \rightarrow 0} \sup _{B \in \mathscr{F}}\left\|\int_{A \cap B} X\right\|=0 \text {. }
$$

(and of course $\|X\|_{P}<\infty$ ).

One question is coming up immediately: What about the following possible definition of Pettis uniform integrability:

$$
\lim _{\lambda \rightarrow \infty} \sup _{n \in N} \sup _{B \in \mathscr{F}}\left\|\int_{\left\{B \cap\left\|X_{n}\right\|>\lambda\right\}} X_{n}\right\|=0 .
$$

Certainly this is only meaningful if the sequence $\left(X_{n}\right)_{n \in N}$ consists of strongly measurable functions (only then $\left\{\left\|X_{n}\right\|>\lambda\right\}$ is surely in $\mathscr{F}$ ). In this case, what is the relation with Definition 2.2.1? We only have the following easy result:

THEOREM 2.2.2. Let $\left(X_{n}\right)_{n \in N}$ be a sequence consisting of strongly measurable Pettis integrable fuunctions. Suppose

$$
\lim _{\lambda \rightarrow \infty} \sup _{n \in N} P\left(\left\|X_{n}\right\|>\lambda\right)=0
$$

Then the following two assertions are equivalent:

(i) $\left(X_{n}\right)_{n \in N}$ is Pettis uniformly integrable.

(ii) $\lim _{\lambda \rightarrow \infty} \sup _{n \in N} \sup _{B \in \mathscr{F}}\left\|\int_{B \cap\left\{\left\|X_{n}\right\|>\lambda\right\}} X_{n}\right\|=0$.

Proof. (i) $\Rightarrow$ (ii). Is done much in the same way as in the case of (Bochner) uniform integrability and hence the proof is omitted. We do not use condition (1) here.

(ii) $\Rightarrow$ (i). Is obvious, using condition (1).

Theorem 2.2.2 is an extension of a remark in Bru-Heinich [B-H] proving that a strongly measurable function $X$ is Pettis integrable if

$$
\lim _{\lambda \rightarrow \infty} \sup _{B \in \mathscr{F}}\left\|\int_{B \cap\{\|X\|>\lambda\}} X\right\|=0 .
$$


Indeed, since $\|X\|$ is measurable we always have here $\lim _{\lambda \rightarrow \infty} P(\|X\|>\lambda)$ $=0$. To indicate some cases in which (1) is satisfied we prove the following

THEOREM 2.2.3. Consider the following assertions for an adapted sequence $\left(X_{n}, \mathscr{F}_{n}\right)_{n \in N}$ consisting of strongly measurable Pettis integrable functions:

(a) $\left(X_{n}, \mathscr{F}_{n}\right)_{n \in N}$ is an $L_{E}^{1}$-bounded martingale,

(a') $\left(X_{n}, \mathscr{F}_{n}\right)_{n \in N}$ is of class $(B)$; i.e. $\sup _{\tau \in T} \int_{\Omega}\left\|X_{\tau}\right\|<\infty$,

(b) $\lim _{\lambda \rightarrow \infty} P\left(\sup _{n \in N}\left\|X_{n}\right\|>\lambda\right)=0$,

(c) $\lim _{\lambda \rightarrow \infty} \sup _{\tau \in T} P\left(\left\|X_{\tau}\right\|>\lambda\right)=0$,

(c') $\lim _{\lambda \rightarrow \infty} \sup _{n \in N} P\left(\left\|X_{n}\right\|>\lambda\right)=0$,

(d) $\lim _{\tau \in T} P\left(\left\|X_{\tau}\right\|>\tau\right)=0$,

(d') $\lim _{n \in N} P\left(\left\|X_{n}\right\|>n\right)=0$.

Then we have

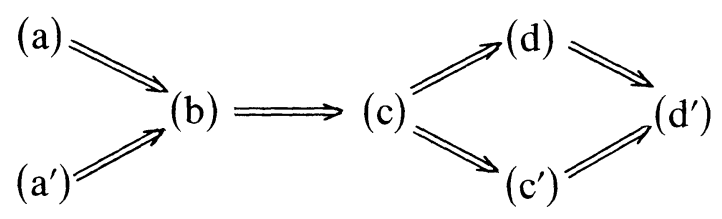

Proof. (a) $\Rightarrow$ (b) and $\left(\mathrm{a}^{\prime}\right) \Rightarrow$ (b) follow from the classical maximal inequality for adapted sequences of Bochner integrable functions.

(b) $\Rightarrow(c) \Rightarrow\left(c^{\prime}\right)$ is trivial.

(c) $\Rightarrow$ (d). For each $\varepsilon>0$, choose $\lambda_{0} \in N$ such that if $\lambda \geq \lambda_{0}$, $\sup _{\tau \in T} P\left(\left\|X_{\tau}\right\|>\lambda\right) \leq \varepsilon$.

For each $\tau \geq \lambda_{0}, \tau \in T$ we so have

$$
P\left(\left\|X_{\tau}\right\|>\tau\right) \leq P\left(\left\|X_{\tau}\right\|>\lambda_{0}\right) \leq \sup _{\tau \in T} P\left(\left\|X_{\tau}\right\|>\lambda_{0}\right) \leq \varepsilon .
$$

$\left(c^{\prime}\right) \Rightarrow\left(d^{\prime}\right)$ is similar

(d) $\Rightarrow\left(d^{\prime}\right)$ is true since $N$ is cofinal in $T$.

EXAMPLES 2.2.4.

1. For any function $X \in P_{E}$ which is strongly measurable we have

$$
\lim _{\lambda \rightarrow \infty} P(\|X\|>\lambda)=0 .
$$

2. Let $E$ be a $\sigma$-Dedekind complete Banach lattice and suppose $\left(X_{n}, \mathscr{F}_{n}\right)$ is an adapted sequence of strongly measurable Pettis integrable functions. 

Then

Suppose also that, for each $n \in N, 0 \leq X_{n} \leq Y$, a.e. where $Y \in P_{E}$.

$$
\lim _{\lambda \rightarrow \infty} P\left(\sup _{n \in N}\left\|X_{n}\right\|>\lambda\right)=0
$$

Proof. Since $E$ is $\sigma$-Dedekind complete, $\sup _{n \in N} X_{n}(\omega) \in E$ exists, a.e. Hence

$$
0 \leq X_{n} \leq \sup _{n \in N} X_{n} \leq Y, \text { a.e. }
$$

Now $\sup _{n \in N} X_{n}$ is strongly measurable and $Y \in P_{E}$. Hence by $[\mathbf{J}] \mathrm{pp}$. 19-20, Theorem 2.10, $\sup _{n \in N} X_{n} \in P_{E}$. In other words we may and do suppose that $Y$ itself is strongly measurable. So $\|Y\|$ is measurable. Furthermore

$$
\sup _{n \in N}\left\|X_{n}\right\| \leq\|Y\|, \quad \text { a.e. }
$$

So (the inclusion is valid a.e.), for each $\lambda>0$

$$
\left\{\sup _{n \in N}\left\|X_{n}\right\|>\lambda\right\} \subset\{\|Y\|>\lambda\} \text {. }
$$

Since $\|Y\|$ is measurable it follows that $\lim _{\lambda \rightarrow \infty} P(\|Y\|>\lambda)=0$. Hence also

$$
\lim _{\lambda \rightarrow \infty} P\left(\sup _{n \in N}\left\|X_{n}\right\|>\lambda\right)=0
$$

3. Pointwise convergence of martingales. In this section it is our purpose to extend Chatterji's martingale convergence theorem $([\mathbf{C}])$ stating that in every Banach space $E$ with (RNP), each $L_{E}^{1}$-bounded martingale converges strongly a.e. to an integrable function. As was done already in $\left[\mathbf{U}_{2}\right]$, the $L_{E}^{1}$-boundedness condition was weakened as to be usable in the case of Pettis integrable functions (i.e. his condition (ii) in Theorem 3.1, p. 375 in $\left[\mathbf{U}_{2}\right]$, or see $\$ 1$ ).

Of the same nature is our extension: instead of $L_{E}^{1}$-boundedness, a notion of " $\sigma-L_{E}^{1}$-boundedness" is required (see condition (ii) in the theorem below) which is natural since Pettis integrals are of $\sigma$-bounded variation $\left(\left[\mathbf{M u} \mathbf{u}_{\mathbf{1}}\right]\right)$.

THEOREM 3.1. Let $E$ be a Banach space such that $c_{0}$ is not isomorphic to a subspace of $E$ (denoted $\left.c_{0} \not t E\right)$. Let $\left(X_{n}, \mathscr{F}_{n}\right)_{n \in N}$ be a martingale 
consisting of strongly measurable Pettis integrable functions such that

(i) $\sup _{n \in N}\left\|X_{n}\right\|_{P}<\infty$ (i.e. condition (i) in Theorem 3.1 in $\left[\mathbf{U}_{2}\right]$ )

(ii) There is a disjoint sequence $\left(A_{n}\right)_{n \in N}$ in $\bigcup_{n \in N} \mathscr{F}_{n}$ such that for each $m \in N$

$$
\sup _{n \in N} \int_{A_{m}}\left\|X_{n}\right\|<\infty .
$$

(iii) For each $\varepsilon>0$ there is a weakly compact set $K \subset E$ such that for each $\varepsilon>0$ there exists $n_{0} \in N$ and a set $A_{0} \in \mathscr{N}_{n_{0}}$ with $P\left(\Omega \backslash A_{0}\right)<\varepsilon$ such that $n \geq n_{0}$ implies $\int_{A} X_{n} \in P(A) K+\delta B_{E}$ for all $A \subset A_{0}, A \in \mathscr{F}_{n}$, (i.e. condition (iii) in Theorem 3.1 in $\left[\mathrm{U}_{2}\right]$ ). (This condition may in fact be localized on each $A_{m}$.)

Then there exists $X_{\infty} \in P_{E}$ (of course strongly measurable) such that $\left(X_{n}\right)_{n \in N}$ converges strongly a.e. to $X_{\infty}$.

Proof. For each $m \in N$, let $n(m) \in N$ be such that $A_{m} \in \mathscr{F}_{n(m)}$. Then

$$
\left(\left.X_{n}\right|_{A_{m}},\left.\mathscr{F}_{n}\right|_{A_{m}}\right)_{n \geq n(m)}
$$

is a martingale in $L_{E}^{1}\left(A_{m}\right)$ satisfying the conditions of [ $\left.\mathbf{U}_{\mathbf{1}}\right]$, Theorem 4 . So there exists $X_{\infty}^{m} \in L_{E}^{1}\left(A_{m}\right)$ such that $\left(\left.X_{n}\right|_{A_{m}}\right)_{n \in N}$ converges a.e. to $X_{\infty}^{m}$ for each $m \in N$. Put

$$
X_{\infty}=\sum_{m=1}^{\infty} X_{\infty}^{m} \chi_{A_{m}} .
$$

Obviously $\left(X_{n}\right)_{n \in N}$ converges strongly a.e. to $X_{\infty}$.

Furthermore $X_{\infty} \in P_{E}$. Indeed, from (i) it follows that for every $x^{\prime} \in E^{\prime},\left(\left\langle x^{\prime}, X_{n}\right\rangle\right)_{n \in N}$ is an $L^{1}$-bounded martingale so a.e. convergent to a function in $L^{1}$. So $X_{\infty}$ is scalarly integrable. Since $c_{0} \not \subset E$ it now follows from $\left[\mathbf{T}_{1}\right]$ (see also $[\mathbf{B}-\mathbf{H}],[\mathbf{J}]$ ), since $X_{\infty}$ is strongly measurable, that $X_{\infty} \in P_{E}$.

COROLlaRY 3.2. Let $E$ have (RNP). Then every martingale $\left(X_{n}, \mathscr{F}_{n}\right)_{n \in N}$ consisting of strongly measurable Pettis integrable functions such that

(i) $\sup _{n \in N}\left\|X_{n}\right\|_{P}<\infty$.

(ii) There is a disjoint sequence $\left(A_{n}\right)_{n \in N}$ in $\bigcup_{n \in N} \mathscr{F}_{n}$ such that for each $m \in N$

$$
\sup _{n \in N} \int_{A_{m}}\left\|X_{n}\right\|<\infty
$$

is strongly converging a.e. to a Pettis integrable function. 
Proof. Indeed, for each $m \in N$, the martingale $\left(\left.X_{n}\right|_{A_{m}},\left.\mathscr{F}_{n}\right|_{A_{m}}\right)_{n \geq n(m)}$ ( $n(m)$ was defined in the previous theorem) is $L_{E}^{1}\left(A_{m}\right)$-bounded, with $E$ (RNP). An appeal to Chatterji's theorem [C] now delivers $X_{\infty}^{m}$ as in the previous proof. The rest is now the same as in this proof since $c_{0} \& E$.

This corollary is a meaningful extension of Chatterji's theorem (even in case $X_{n} \in L_{E}^{1}$ for each $n \in N$, it does not reduce to Chatterji's theorem).

We shall use the corollary later in this paper.

4. Pettis convergence of amarts. It is our purpose in this section to generalize and reprove Musial's result on Pettis convergence of martingales (or amarts). This is done by first proving a Riesz decomposition theorem in this setting, generalizing the Riesz decomposition of Edgar-Sucheston [E-S].

THEOREM 4.1. Let E be a Banach space with $(W R N P)$ and $\left(X_{n}, \mathscr{F}_{n}\right)_{n \in N}$ be an amart in $P_{E}$ (not necessarily consisting of strongly measurable functions).

Suppose there is a disjoint sequence $\left(A_{k}\right)_{k \in N}$ in $\mathscr{F}_{1}$ and a strictly increasing sequence $\left(m_{n}\right)_{n \in N}$ in $N$ such that

$$
\sup _{n \in N} \int_{A_{k}}\left\|X_{m_{n}}\right\|<\infty
$$

for each $k \in N$. Then there is a unique decomposition

$$
X_{n}=Y_{n}+Z_{n}
$$

where $\left(Y_{n}, \mathscr{F}_{n}\right)_{n \in N}$ is a martingale in $P_{E}$ such that

$$
\sup _{n \in N} \int_{A_{k}}\left\|Y_{n}\right\|<\infty
$$

for each $k \in N$ and $\left(Z_{n}, \mathscr{F}_{n}\right)_{n \in N}$ is an adapted sequence such that

$$
\lim _{\tau \in T}\left\|Z_{\tau}\right\|_{P}=0
$$

Proof. From the definition of an amart we can see (in the same way as in the case of Bochner integrable functions-[B], Theorem 1, p. 279) that, for each $i \in N$

$$
\mu_{i}(A)=: \lim _{n \rightarrow \infty} \int_{A} X_{m_{n}}
$$


exists for each $A \in \mathscr{F}_{i}$. It is easy to check that $\mu_{i}$ is of $\sigma$-bounded variation w.r.t. (the sets $\left(A_{k}\right)_{k \in N}$ suffice) and that $\mu_{i} \ll P$. Using (WRNP) this yields $Y_{i} \in P_{E}$ such that

$$
\int_{A} Y_{t}=\mu_{t}(A)
$$

for each $A \in \mathscr{F}_{i}$ and each $i \in N$.

Now $\left(Y_{n}, \mathscr{F}_{n}\right)_{n \in N}$ is easily seen to be a martingale and furthermore since $\sup _{n \in N}\left|\mu_{n}\right|_{A_{k}} \mid\left(A_{k}\right)<\infty$, for each $k \in N$, we see that

$$
\sup _{n \in N} \int_{A_{k}}\left\|Y_{n}\right\|<\infty
$$

for each $k \in N$. Furthermore, for each $\sigma \in T$ and each $A \in \mathscr{F}_{\sigma}$

$$
\left\|\int_{A}\left(Y_{\sigma}-X_{\sigma}\right)\right\|=\left\|\lim _{\substack{n \rightarrow \infty \\ m_{n} \geq 0}} \int_{A} X_{m_{n}}-\int_{A} X_{\sigma}\right\| .
$$

So

$$
\begin{aligned}
\sup _{A \in \mathscr{F}_{\sigma}}\left\|\int_{A}\left(Y_{\sigma}-X_{\sigma}\right)\right\| & =\sup _{A \in \mathscr{F}_{\sigma}}\left\|\lim _{\substack{n \rightarrow \infty \\
m_{n} \geq \sigma}} \int_{A} X_{m_{n}}-\int_{A} X_{\sigma}\right\| \\
& \leq \sup _{m_{n} \geq \sigma} \sup _{A \in \mathscr{F}_{\sigma}}\left\|\int_{A} X_{m_{n}}-\int_{A} X_{\sigma}\right\| .
\end{aligned}
$$

The term on the right-hand side goes to zero for $\sigma$ going through $T$, again using [B] Theorem 1, p. 279, extended to our case. Hence

$$
\lim _{\sigma \in T}\left\|Z_{\sigma}\right\|_{P}=\lim _{\sigma \in T}\left\|Y_{\sigma}-X_{\sigma}\right\|_{P}=0 .
$$

The uniqueness of the decomposition is proved in exactly the same way as in the Bochner integrable case: suppose

$$
X_{n}=Y_{n}+Z_{n}=Y_{n}^{\prime}+Z_{n}^{\prime}
$$

where $\left(Y_{n}, \mathscr{F}_{n}\right)_{n \in N}$ and $\left(Y_{n}^{\prime}, \mathscr{F}_{n}\right)_{n \in N}$ are martingales and where $\lim _{\tau \in T}\left\|Z_{\tau}\right\|_{P}=\lim _{\tau \in T}\left\|Z_{\tau}^{\prime}\right\|_{P}=0$.

Then, for each $A \in \bigcup_{n} \mathscr{F}_{n}$

$$
\lim _{n \rightarrow \infty} \int_{A}\left(Z_{n}-Z_{n}^{\prime}\right)=0 .
$$


But $Y_{n}^{\prime}-Y_{n}=Z_{n}^{\prime}-Z_{n}$ forms also a martingale. So, for each $m \in N$ and $A \in \mathscr{F}_{m}$

$$
\lim _{n \rightarrow \infty} \int_{A}\left(Z_{n}-Z_{n}^{\prime}\right)=\int_{A}\left(Z_{m}-Z_{m}^{\prime}\right)=0 .
$$

So $Z_{m}=Z_{m}^{\prime}$, a.e. and also $Y_{m}=Y_{m}^{\prime}$, a.e. for each $m \in N$.

COROLlary 4.2. Let $E$ have (WRNP) and $\left(X_{n}, \mathscr{F}_{n}\right)_{n \in N}$ be an amart in $P_{E}$ such that there is a disjoint sequence $\left(A_{k}\right)_{k \in N}$ in $\mathscr{F}_{1}$ and a strictly increasing sequence $\left(m_{n}\right)_{n \in N}$ in $N$ such that

$$
\sup _{n \in N} \int_{A_{k}}\left\|X_{m_{n}}\right\|<\infty
$$

for each $k \in N$. Suppose that $\left(X_{n}\right)_{n \in N}$ is Pettis uniformly integrable. Then there exists a function $X_{\infty} \in P_{E}$ such that $\lim _{n \rightarrow \infty}\left\|X_{n}-X_{\infty}\right\|_{P}=0$.

Proof. Using Theorem 4.1 above it suffices to prove the result for the martingale $\left(Y_{n}, \mathscr{F}_{n}\right)_{n \in N}$ constructed in the above proof. Obviously, since $\lim _{\sigma \in T}\left\|Z_{\sigma}\right\|_{P}=0,\left(Y_{n}\right)_{n \in N}$ is Pettis uniformly integrable too.

The limit measure $\mu(A)=\lim _{n \rightarrow \infty} \int_{A} Y_{n}$ exists on $\bigcup_{n} \mathscr{F}_{n}$ and, due to the Pettis uniform integrability, on $\mathscr{F}_{\infty}=\sigma\left(\bigcup_{n} \mathscr{F}_{n}\right)$. It now follows that $\mu$ is of $\sigma$-bounded variation on $\mathscr{F}_{\infty}$ and that $\mu \ll P$.

So, the (WRNP) of $E$ implies that $Y_{\infty} \in P_{E}$ exists such that

$$
\int_{A} Y_{\infty}=\mu(A)
$$

for each $A \in \mathscr{F}_{\infty}$. We have $\lim _{n \rightarrow \infty}\left\|Y_{n}-Y_{\infty}\right\|_{P}=0$ since $Y_{n}=E^{\mathscr{F}_{n}} Y_{\infty}$ for each $n \in N$. This is seen in the next lemma.

LEMMA 4.2.1. Let $E$ have (WRNP). Let $Y \in P_{E}$ be such that w.r.t. $\left(\mathscr{F}_{n}\right)_{n \in N}, E^{\mathscr{F}_{n}} Y \in P_{E}$ exists, for each $n \in N$. Then $\lim _{n \rightarrow \infty}\left\|Y-E^{\mathscr{F}_{n}} Y\right\|_{P}=$ 0 .

Proof. If $Y$ is a simple function, the result is certainly true (and even $\lim _{n \rightarrow \infty}\left\|Y-E^{\mathscr{F}} Y\right\|_{1}=0$ in this case!).

The general case follows, using a $\|\cdot\|_{P}$ density argument. Simple functions are indeed dense in $P_{E}$ since $E$ has (WRNP), see [ $\left[\mathbf{M u}_{3}\right]$, p. 330, Theorem 1. 
REMARKS 4.2.2. (1) In the above lemma we in fact only used that $E$ has the so-called "Pettis Compactness Property" i.e. In $E$ has every Pettis integral norm relatively compact range.

(2) Corollary 4.2 extends the main result in $\left[\mathbf{M u}_{3}\right]$ (Theorem 5, p. 334) and gives a simpler proof for it. Indeed we did not use Proposition 2 in $\left[\mathbf{M u}_{3}\right]$.

The next corollary belongs to this section but is in fact only a corollary of Corollary 3.2.

COROLlary 4.3. Let $E$ have $(R N P)$ and $\left(X_{n}, \mathscr{F}_{n}\right)_{n \in N}$ be a martingale in $P_{E}$ such that there is a disjoint sequence $\left(A_{k}\right)_{k \in N}$ in $\bigcup_{n} \mathscr{F}_{n}$ such that for each $k \in N$

$$
\sup _{n \in N} \int_{A_{k}}\left\|X_{n}\right\|<\infty
$$

and such that $\left(X_{n}\right)_{n \in N}$ is Pettis uniformly integrable. Then there is a function $X_{\infty} \in P_{E}$ such that $\lim _{n \rightarrow \infty}\left\|X_{n}-X_{\infty}\right\|_{P}=0$.

Proof. Since $E$ has (RNP) and since we are dealing with $\|\cdot\|_{P^{-}}$ convergence we may suppose (see $[\mathbf{J}]$, p. 129) that every $X_{n}$ is strongly measurable (cf. the discussion in $\S 1$ concerning Musiał's theorem). From Corollary 3.2 we have $X_{\infty} \in P_{E}$ such that $\lim _{n \rightarrow \infty} X=X_{\infty}$, a.e. So, the Pettis uniform integrability of $\left(X_{n}\right)_{n \in N}$ finishes the proof.

5. Weak convergence of weak sequential amarts. It is our purpose to extend the theorem of Brunel-Sucheston $[\mathbf{B}-\mathbf{S}]$ on weak convergence a.e. of weak sequential amarts to the case of strongly measurble Pettis integrable functions. First a definition.

Definition 5.1. Let $\left(X_{n}, \mathscr{F}_{n}\right)_{n \in N}$ be an adapted sequence in $P_{E}$. We say that $\left(X_{n}, \mathscr{F}_{n}\right)_{n \in N}$ is a weak sequential amart (abbreviated (WS) amart) if for every increasing sequence $\left(\tau_{n}\right)_{n \in N}$ in $T$ the sequence $\left(\int_{\Omega} X_{\tau_{n}}\right)_{n \in N}$ converges weakly in $E$.

The same proof as in the case of Bochner integrable functions shows that every amart is a (WS) amart.

In $[\mathbf{B}-\mathbf{S}]$, Brunel-Sucheston proved the following extension of a result on amarts of Chacon-Sucheston [C-S] to (WS) amarts.

THEOREM 5.2 (Brunel-Sucheston). Let $E$ be a Banach space with $(R N P)$ and with separable dual. Let $\left(X_{n}, F_{n}\right)_{n \in N}$ be a (WS) amart of class 
(B), i.e.

$$
\sup _{\tau \in T} \int_{\omega}\left\|X_{\tau}\right\|<\infty
$$

Then there exists $X_{\infty} \in L_{E}^{1}$ such that $X_{n}$ converges to $X_{\infty}$, weakly a.e.

For (WS) amarts consisting of strongly measurable Pettis integrable functions we can prove

THEOREM 5.3. Let $E$ and $E^{\prime}$ have (RNP). Let $\left(X_{n}, \mathscr{F}_{n}\right)_{n \in N}$ be a (WS) amart in $P_{E}$, consisting of strongly measurable functions. Suppose that:

(i) $\lim _{\lambda \rightarrow \infty} P\left(\sup _{n \in N}\left\|X_{n}\right\|>\lambda\right)=0$

(ii) $\sup _{\tau \in T} \int_{\left\{\left\|X_{\tau}\right\| \leq \tau\right\}}\left\|X_{\tau}\right\|<\infty$

(iii) $\left(X_{\tau}\right)_{\tau \in T}$ is Pettis uniformly integrable.

Then there is a $X_{\infty} \in L_{E}^{1}$ such that $\left(X_{n}\right)_{n \in N}$ converges weakly a.e. to $X_{\infty}$

Proof. For each $n \in N$, define

$$
Y_{n}=X_{n} \chi_{\left\{\left\|X_{n}\right\| \leq n\right\}} .
$$

Then $\left(Y_{n}, \mathscr{F}_{n}\right)_{n \in N}$ is an adapted sequence in $L_{E}^{1}$, due to the fact that every $\left\|X_{n}\right\|$ is measurable. $\left(Y_{n}, \mathscr{F}_{n}\right)_{n \in N}$ is furthermore a (WS) amart. Indeed, let $\left(\tau_{n}\right)_{n \in N}$ be any increasing sequence in $T$. We may assume that $\lim _{n \rightarrow \infty} \tau_{n}$ $=\infty$. Now

$$
\int_{\omega}\left(X_{\tau_{n}}-Y_{\tau_{n}}\right)=\int_{\left\{\left\|X_{\tau_{n}}\right\|>\tau_{n}\right\}} X_{\tau_{n}}
$$

Since $\lim _{\lambda \rightarrow \infty} P\left(\sup _{n \in N}\left\|X_{n}\right\|>\lambda\right)=0$ it follows that

$$
\lim _{n \rightarrow \infty} P\left(\left\|X_{\tau_{n}}\right\|>\tau_{n}\right)=0
$$

(even if $\left(\tau_{n}\right)_{n \in N}$ is not cofinal in $T$ ). Hence, using (iii) one sees easily that

$$
\lim _{n \rightarrow \infty} \sup _{A \in \mathscr{F}}\left\|\int_{A \cap\left\{\left\|X_{\tau_{n}}\right\|>\tau_{n}\right\}} X_{\tau_{n}}\right\|=0 .
$$

So

$$
\lim _{n \rightarrow \infty}\left\|\int_{\left\{\left\|X_{\tau_{n}}\right\|>\tau_{n}\right\}} X_{\tau_{n}}\right\|=0
$$


Since $\left(X_{n}, \mathscr{F}_{n}\right)_{n \in N}$ is a (WS) amart, there is an $x_{0} \in E$ such that

$$
\underset{n \rightarrow \infty}{\text { weak-lim }} \int_{\Omega} X_{\tau_{n}}=x_{0} .
$$

So, also weak- $\lim _{n \rightarrow \infty} \int_{\Omega} Y_{\tau_{n}}=x_{0}$.

Using (ii), $\left(Y_{n}, \mathscr{F}_{n}\right)_{n \in N}$ is of class $(B)$.

Hence by Theorem 5.2, there is $Y_{\infty} \in L_{E}^{1}$ such that weak- $\lim _{n \rightarrow \infty} Y_{n}=$ $Y_{\infty}$, a.e. Now

$$
\underset{n}{\lim \sup }\left\|X_{n}-Y_{n}\right\|=\limsup _{n}\left\|X_{n}\right\| \chi_{\left\{\left\|X_{n}\right\|>n\right\}} .
$$

Since $\left\{\left\|X_{n}\right\|>n\right\} \subset\left\{\sup _{n}\left\|X_{n}\right\|>\lambda\right\}$ if $n \geq \lambda$ and by (i) we have now that $\lim \sup _{n}\left\|X_{n}-Y_{n}\right\|=0$. Consequently $\left(X_{n}\right)_{n \in N}$ converges weakly a.e. to $Y_{\infty}$.

REMARK 5.4. From (ii) we knew already in advance that whenever $\left(X_{n}\right)_{n \in N}$ converges weakly a.e., the limit must be Bochner integrable. Indeed:

$$
\sup _{\tau \in T} \int_{\left\{\left\|X_{\tau}\right\| \leq \tau\right\}}\left\|X_{\tau}\right\| \geq \limsup _{\tau \in T} \int_{\left\{\left\|X_{\tau}\right\| \leq \tau\right\}}\left\|X_{\tau}\right\|=\int_{\Omega} \limsup _{n}\left\|X_{n}\right\| \chi_{\left\{\left\|X_{n}\right\| \leq n\right\}}
$$

(see [B-E], [Ed]).

Now, if we suppose weak convergence a.e. of $\left(X_{n}\right)_{n \in N}$, we have that $\left(X_{n}(\omega)\right)_{n \in N}$ is weakly, hence strongly bounded a.e. So

$$
\int_{\Omega} \limsup _{n}\left\|X_{n}\right\| \chi_{\left\{\left\|X_{n}\right\| \leq n\right\}}=\int_{\Omega} \limsup _{n}\left\|X_{n}\right\|
$$

So (ii) implies

$$
\int_{\Omega}\left\|\underset{n \rightarrow \infty}{\operatorname{weak} \lim } X_{n}\right\| \leq \int_{\Omega} \liminf _{n}\left\|X_{n}\right\| \leq \int_{\Omega} \limsup _{n}\left\|X_{n}\right\|<\infty .
$$

\section{Strong convergence of pramarts.}

Definition 6.1. Let $\left(X_{n}, \mathscr{F}_{n}\right)_{n \in N}$ be an adapted sequence in $P_{E}$. Suppose that for every $\sigma, \tau \in T$ with $\sigma \leq \tau$ the conditional expectations $E^{\mathscr{F}_{\sigma}} X_{\epsilon} \in P_{E}$ exist. 
$\left(X_{n}, \mathscr{F}_{n}\right)_{n \in N}$ is called a pramart if for every $\varepsilon>0$ there exists $\sigma_{0} \in T$ such that $\tau \geq \sigma \geq \sigma_{0}, \tau, \sigma \in T$ imply

$$
P\left(\left\|E^{\mathscr{F _ { \sigma }}} X_{\tau}-X_{\sigma}\right\| \geq \varepsilon\right) \leq \varepsilon .
$$

In [M-S], Millet-Sucheston prove

Theorem 6.2 (Millet-Sucheston). Let E be a Banach space with (RNP). Then every pramart $\left(X_{n}, \mathscr{F}_{n}\right)_{n \in N}$ in $L_{E}^{1}$ such that $\sup _{\tau \in T} \int_{\Omega}\left\|X_{\tau}\right\|<\infty$ is convergent strongly, a.e. (to a Bochner integrable function).

We can show now the following extension of Theorem 6.2

THEOREM 6.3. Let $E$ be a Banach space with $(R N P)$. Let $\left(X_{n}, \mathscr{F}_{n}\right)_{n \in N}$ be a pramart consisting of strongly measurable Pettis integrable functions. Suppose that

(i) $\lim _{n \rightarrow \infty} P\left(\sup _{n \in N}\left\|X_{n}\right\|>\lambda\right)=0$

(ii) $\sup _{\tau \in T} \int_{\left\{\left\|X_{\tau}\right\|<\tau\right\}}\left\|X_{\tau}\right\|<\infty$.

Then there is a $X_{\infty} \in L_{E}^{1}$ such that $\left(X_{n}\right)_{n \in N}$ converges strongly a.e. to $X_{\infty}$.

Proof. We start in the same way as in the proof of Theorem 5.3. Indeed we also apply Theorem 6.2 on $\left(Y_{n}, \mathscr{F}_{n}\right)_{n \in N}$ where $Y_{n}=X_{n} \chi_{\left\{\left\|X_{n}\right\| \leq n\right\}}$ for every $n \in N$. $\left(Y_{n}, \mathscr{F}_{n}\right)_{n \in N}$ is indeed a pramart due to (i).

Since, as in Theorem 5.3 we also have

$$
\limsup _{n}\left\|X_{n}-Y_{n}\right\|=0
$$

it follows that $\left(X_{n}\right)_{n \in N}$ converges strongly a.e. to $\lim _{n \rightarrow \infty} Y_{n} \in L_{E}^{1}$.

REMARKS 6.4.

(1) We have not used condition (iii) of Theorem 5.3 in the proof of the above theorem.

(2) In Theorem 6.2, (i) does not appear but is of course implied by the condition

$$
\sup _{\tau \in T} \int_{\Omega}\left\|X_{\tau}\right\|<\infty
$$

which appears there. So even if $X_{n} \in L_{E}^{1}$ for each $n \in N$, Theorem 6.3 is an extension of Theorem 6.2.

Acknowledgement. I thank Dr. Jeurnink (University of Nijmegen, the Netherlands) for sending me a copy of his Ph.D. 


\section{REFERENCES}

[B] A. Bellow, Several stability properties of the class of asymptotic martingales, Z. Wahrscheinlichkeitstheorie und verw. Geb., 37(1977), 275-290.

[B-E] A. Bellow et L. Egghe, Inégalités de Fatou généralisées, c. R. Acad. Sci. Paris, t. 292, série I, (1981), 847-850.

[B-H] B. Bru et H. Heinich, Sur l'espérance des variables aléatoires vectorielles, preprint 1980.

[B-S] A. Brunel et L. Sucheston, Sur les amarts faibles à valeurs vectorielles, C. R. Acad. Sci. Paris, t. 282, série A, (1976), 1011-1014.

[C-S] R. V. Chacon and L. Sucheston, On convergence of vector-valued asymptotic martingales, Z. Wahrscheinlichkeitstheorie und verw. Geb., 33 (1975), 55-59.

[C] S. D. Chatterji, Martingale convergence and the Radon-Nikodym theorem, Math. Scand., 22 (1968), 21-44.

[Die] J. Diestel, Geometry of Banach Spaces-Selected Topics, Lecture Notes in Math., 485, Springer-Verlag, 1975.

[D-U] J. Diestel and J. J. Uhl Jr., Vector measures, Amer. Math. Soc.-mathematical surveys, 15 (1977).

[Din] N. Dinculeanu, Vector measures, Pergamon Press, 95 (1967).

[D-R] A. Dvoretzky and C. A. Rogers, Absolute and unconditional convergence in normed linear spaces, Proc. Nat. Acad. Sci. USA, 36 (1950), 192-197.

[D-S] N. Dunford and J. T. Shcwartz, Linear Operators, Part I (General Theory). Interscience Publishers, 1957.

[Ed] G. A. Edgar, Uniform semiamarts, Ann. Inst. H. Poincaré t. 15, sect. b, (1979), 197-203.

[E-S] G. A. Edgar and L. Sucheston, The Riesz-decomposition for vector-valued amarts, Z. Wahrscheinlichkeitstheorie und verw. Gebiete, 36 (1976), 85-92.

[Eg] L. Egghe, Stopping time techniques for analysts and probabilists, Lond. Math. Soc. Lect. Notes Ser., Cambridge Univ. Press (to appear).

[H] H. Heinich, Espérance conditionnelle pour les fonctions vectorielles, C. R. Acad. Sci. Paris, t. 276, série A, (1973), 935-938.

[J-K] L. Janicka and N. Kalton, Vector measures of infinite variation, Bull. de l'Acad. Pol. des Sci., 25 (1977), 239-241.

[J] G. Jeurnink, Integration of functions with values in a Banach space, Ph.D. Univ. of Nijmegen (the Netherlands), 1982.

[Me] M. Métivier, Martingales à valueurs vectorielles. Applications à la dérivation des mesures vectorielles, Ann. Inst. Fourier, 17 (1967), 175-208.

[M-S] A. Millet and L. Sucheston, Characterizations of Vitali conditions with overlap in terms of convergence of classes of amarts, Canad. J. Math., 31 (1979), 1033-1046.

$\left[\mathrm{Mu}_{1}\right]$ K. Musial, The weak Radon-Nikodym property in Banach spaces, preprint, 1976.

$\left[\mathrm{Mu}_{2}\right]$, The weak Radon-Nikodym property in Banach spaces, Studia Math., 64 (1979), 151-173.

$\left[\mathrm{Mu}_{3}\right]$ Martingales of Pettis Integrable Functions, Proc. Conf. Measure Theory, Oberwolfach 1979, Lect. Notes in Math. 794, Springer Verlag, (1980), 324-339.

[P] B. J. Pettis, On integration in vector spaces, Trans. Amer. Math. Soc., 44 (1938), 277-304.

[R] V. I. Rybakov, On the conditional expectations of Pettis integrable functions (in Russian), Matiematiceskije Zamietki, 10 (1971), 565-570.

[S] M. Slaby, Convergence of submartingales and amarts in Banach lattices, Bull. Acad. Pol. Sci., sér. Sc. Math., 30 (1982), 291-299. 
$\left[\mathrm{T}_{1}\right] \quad \mathrm{E}$. Thomas, L'intégration par rapport à une mesure de Radon vectorielle, Ann. Inst. Fourier, 20 (1970), 55-191.

$\left[\mathrm{T}_{2}\right] \ldots$ the Lebesgue-Nykodym theorem for vector valued Radon measures, Mem. Amer. Math. Soc., 139 (1974).

$\left[\mathrm{T}_{3}\right]$, On Some Negative Properties of the Pettis-integral (addendum), Lecture Notes in Math., Springer Verlag, 541 (1976), 131.

$\left[\mathrm{U}_{1}\right]$ J. J. Uhl Jr., Applications of Radon-Nikodym theorems to martingale convergence, Trans. Amer. Math. Soc., 145 (1969), 271-285.

$\left[\mathrm{U}_{2}\right] \quad$ Martingales of strongly measurable Pettis integrable functions, Trans. Amer. Math. Soc., 167 (1972), 369-378.

$\left[\mathrm{U}_{3}\right]$, Pettis-mean-convergence of vector valued asymptotic martingales, $\mathrm{Z}$. Wahrscheinlichkeitstheorie und verw. Gebiete, 37 (1977), 291-295.

Received November 1, 1982 and in revised form August 18, 1983.

LIMBURgS UNIVERSITAIR CENTRUM

B - 3610 DIEPENBEEK

BELGIUM 


\section{PACIFIC JOURNAL OF MATHEMATICS EDITORS}

\author{
DONALD BABBITT (Managing Editor) \\ University of California \\ Los Angeles, CA 90024 \\ J. DugundJI \\ University of Southern California \\ Los Angeles, CA 90089-1113 \\ R. FINN \\ Stanford University \\ Stanford, CA 94305 \\ HERMANN FLASChKa \\ University of Arizona \\ Tucson, AZ 85721
}

C. C. MOORE

University of California

Berkeley, CA 94720

Arthur Ogus

University of California

Berkeley, CA 94720

Hugo RossI

University of Utah

Salt Lake City, UT 84112

H. SAMELSON

Stanford University

Stanford, CA 94305

ASSOCIATE EDITORS
R. ARENS
E. F. BECKENBACH
B. H. NeUmanN
F. WOLF
K. YosHIDA (1906-1982)

\section{SUPPORTING INSTITUTIONS}
UNIVERSITY OF ARIZONA
UNIVERSITY OF BRITISH COLUMBIA
CALIFORNIA INSTITUTE OF TECHNOLOGY
UNIVERSITY OF CALIFORNIA
MONTANA STATE UNIVERSITY
UNIVERSITY OF NEVADA, RENO
NEW MEXICO STATE UNIVERSITY
OREGON STATE UNIVERSITY

\author{
UNIVERSITY OF OREGON \\ UNIVERSITY OF SOUTHERN CALIFORNIA \\ STANFORD UNIVERSITY \\ UNIVERSITY OF HAWAII \\ UNIVERSITY OF TOKYO \\ UNIVERSITY OF UTAH \\ WASHINGTON STATE UNIVERSITY \\ UNIVERSITY OF WASHINGTON
}

The Supporting Institutions listed above contribute to the cost of publication of this Journal, but they are not owners or publishers and have no responsibility for its content or policies.

Mathematical papers intended for publication in the Pacific Journal of Mathematics should be in typed form or offset-reproduced (not dittoed), double spaced with large margins. Please do not use built up fractions in the text of the manuscript. However, you may use them in the displayed equations. Underline Greek letters in red, German in green, and script in blue. The first paragraph must be capable of being used separately as a synopsis of the entire paper. In particular it should contain no bibliographic references. Please propose a heading for the odd numbered pages of less than 35 characters. Manuscripts, in triplicate, may be sent to any one of the editors. Please classify according to the scheme of Math. Reviews, Index to Vol. 39. Supply name and address of author to whom proofs should be sent. All other communications should be addressed to the managing editor, or Elaine Barth, University of California, Los Angeles, California 90024.

There are page-charges associated with articles appearing in the Pacific Journal of Mathematics. These charges are expected to be paid by the author's University, Government Agency or Company. If the author or authors do not have access to such Institutional support these charges are waived. Single authors will receive 50 free reprints; joint authors will receive a total of 100 free reprints. Additional copies may be obtained at cost in multiples of 50 .

The Pacific Journal of Mathematics is issued monthly as of January 1966. Regular subscription rate: $\$ 190.00$ a year (5 Vols., 10 issues). Special rate: $\$ 66.00$ a year to individual members of supporting institutions.

Subscriptions, orders for numbers issued in the last three calendar years, and changes of address should be sent to Pacific Journal of Mathematics, P.O. Box 969, Carmel Valley, CA 93924, U.S.A. Old back numbers obtainable from Kraus Periodicals Co., Route 100, Millwood, NY 10546.

The Pacific Journal of Mathematics at P.O. Box 969, Carmel Valley, CA 93924 (ISSN 0030-8730) publishes 5 volumes per year. Application to mail at Second-class postage rates is pending at Carmel Valley, California, and additional mailing offices. Postmaster: Send address changes to Pacific Journal of Mathematics, P.O. Box 969, Carmel Valley, CA 93924.

PUBLISHED BY PACIFIC JOURNAL OF MATHEMATICS, A NON-PROFIT CORPORATION

Copyright $\odot 1984$ by Pacific Journal of Mathematics 


\section{Pacific Journal of Mathematics}

Vol. 114, No. $2 \quad$ June, 1984

William Allen Adkins, A Harnack estimate for real normal surface

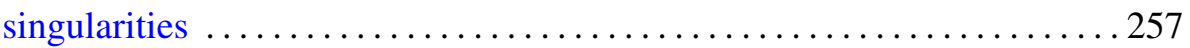

George E. Andrews, Multiple series Rogers-Ramanujan type identities . . . . 267

Didier Arnal, $*$ products and representations of nilpotent groups . . . . . . 285

David Cox and Walter Raymond Parry, Representations associated with

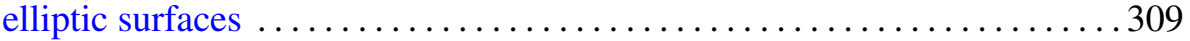

Joanne Marie Dombrowski, Tridiagonal matrix representations of cyclic

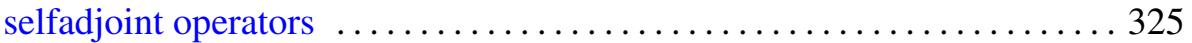

Ronald Dotzel, An Artin relation $(\bmod 2)$ for finite group actions on

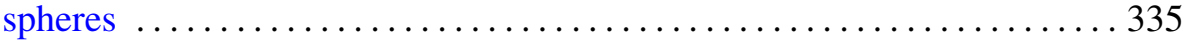

Leo Egghe, Convergence of adapted sequences of Pettis-integrable

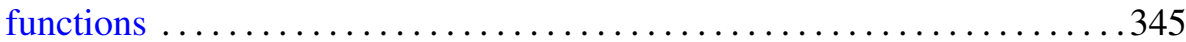

Rebecca A. Herb, Characters of induced representations and weighted

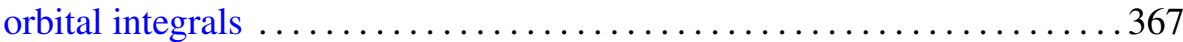

Steven M. Kahn, Cobordism obstructions to fibering manifolds over

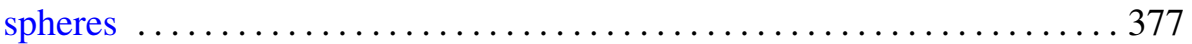

Robert D. Little, Projective space as a branched covering of the sphere with orientable branch set

Claude Schochet, Topological methods for $C^{*}$-algebras. III. Axiomatic

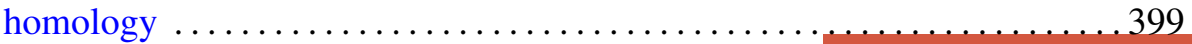

Claude Schochet, Topological methods for $C^{*}$-algebras. IV. $\bmod p$ homology

James M. Stormes, On the $K O$-orientability of complex projective varieties

Josephine Anne Ward, Characterization of homogeneous spaces and their norms 